\title{
Influence of testosterone on mean arterial pressure: A physiological study in male and female normotensive WKY and hypertensive SHR rats
}

\author{
SY Loh, N Salleh \\ Department of Physiology, Faculty of Medicine, University of Malaya, Kuala Lumpur, Malaysia
}

Received: August 30, 2016

Accepted: December 13, 2016

\begin{abstract}
Introduction: Testosterone plays an important role in the blood pressure regulation. However, information with regard to the effect of this hormone on blood pressure in normotensive and hypertensive conditions is limited. Therefore, in this study, the relationship between plasma testosterone level and mean arterial pressure (MAP) was investigated under these conditions. Methods: Normotensive Wistar-Kyoto (WKY) and hypertensive Spontaneous Hypertensive (SHR) male and female rats were gonadectomized with female rats treated with testosterone. Estrous cycle stages of intact female rats of both strains were identified by vaginal smear. Pressure in the carotid artery of anesthetized rats was measured via direct cannulation technique. The blood was withdrawn for plasma testosterone level measurement by enzyme-linked immunosorbent assay. Results: Treatment of ovariectomized female WKY and SHR rats with testosterone for 6-week duration has resulted in MAP to increase $(P<0.05)$. In male WKY and SHR rats, MAP and plasma testosterone levels decreased by orchidectomy $(P<0.05)$. No significant differences in MAP and plasma testosterone levels were observed in intact female WKY and SHR rats between stages of the estrous cycle. Conclusions: The effects seen in testosterone-treated ovariectomized female rats and in orchidectomized male rats suggested that testosterone could play an important role in causing the blood pressure to increase.
\end{abstract}

Keywords: testosterone, gonadectomy, blood pressure, rats, WKY, SHR

\section{Introduction}

The relationship between sex steroids and blood pressure has long been observed $(22,48)$. Gender difference in the blood pressure regulation has long been documented in which men have higher blood pressure as compared with age-matched women before menopause $(10,28$, 42). Studies have shown that testosterone plays a role underlying the gender difference in blood pressure regulation. In men, there was an inverse relationship between blood pressure and plasma testosterone levels $(16,24,47)$. Higher plasma testosterone level is associated with increased blood pressure in males, whereas in females, higher plasma testosterone level has been associated with increased arterial stiffness $(16,45)$. In addition, there is increased risk of hypertension in women possessing high plasma testosterone levels as in polycystic ovary disease $(3,12)$.

The effects of testosterone on blood pressure regulation have been documented in animals. In hypertensive rats, the levels of testosterone correlate with the mean arterial pressure (MAP) $(10,15,23,27,37)$. However, in some studies, testosterone was found to

\footnotetext{
Corresponding author: Naguib Salleh

Department of Physiology, Faculty of Medicine, University of Malaya

50603 Kuala Lumpur, Malaysia

Phone: +603 7967 7532; Fax:+603 7967 4775; E-mail: naguib.salleh@gmail.com
} 
have no effect on the blood pressure $(8,17)$. The effects of sex-steroid hormones on blood pressure in females are far more complex and less well understood as compared with the effects of these hormones on blood pressure in males. Female blood pressure was found to be influenced by estrogen $(9,18,46)$.

To date, there have been limited findings with regard to the effect of testosterone on blood pressure regulation in females. The levels of this hormone were found to fluctuate throughout female reproductive cycle, which suggested that testosterone could influence the blood pressure as there is a mild blood pressure fluctuation observed throughout the cycle $(26,39,40)$. In this study, the effects of testosterone on blood pressure regulation in females were investigated. In addition, the effects of removal of endogenous testosterone on blood pressure regulation in males were also investigated. Furthermore, influence of testosterone on blood pressure throughout the female reproductive cycle was also identified.

\section{Materials and Methods}

\section{Animals}

Eight-week-old male and female Wistar-Kyoto (WKY) and Spontaneous Hypertensive (SHR) rats were maintained in a well-equipped animal facility with a constant 12:12 $\mathrm{h}$ light:dark cycle. All animals had free access to a standard rodent chow diet (Teklad Diet, Rossdoff, Germany) and tap water ad libitum. All procedures were approved by the Institutional Animal Care and Use Committee (IACUC), University of Malaya with ethics number: 2014-05-07/physio/R/NS.

\section{Experimental designs}

In this study, three different experiments were designed and performed independently as follows:

Experiment 1. Male and female WKY and SHR rats were divided into four groups, each group contains five rats. Sham operations, orchidectomies, and ovariectomies were performed at 8 weeks of age in a sterile environment. At 16 weeks of age, animals were subjected for subsequent procedures. Experimental groups were designed as follows:

Group 1: Sham-operated, intact male WKY and SHR rats

Group 2: Orchidectomized (ORX), male WKY and SHR rats

Group 3: Sham-operated, intact female WKY and SHR rats

Group 4: Ovariectomized (OVX), female WKY and SHR rats

Experiment 2. Female WKY and SHR rats were used in this experiment. All animals were subjected for vaginal smear examination at 16 weeks of age. $0.9 \%(\mathrm{w} / \mathrm{v}) \mathrm{NaCl}$ was flushed into the vagina and the fluid was then retrieved. Estrous cycle stage was determined based on the cells found in the vaginal flushing (unstained). The criteria for estrous stages identification were as described by Marcondes et al. (29). All rats were grouped in accordance with the estrous cycle stages, with five animals per group. Four experimental groups per strain were designed as follows:

Group 1: Proestrus stage (Ps)

Group 2: Estrus stage (Es)

Group 3: Metestrus stage (Ms)

Group 4: Diestrus stage (Ds) 
Experiment 3. Male and female WKY and SHR rats were divided into four groups, each group contains five animals. Sham operations, orchidectomies, and ovariectomies were performed at 8 weeks of age. All surgeries were performed under sterile conditions. A group of ovariectomized female rats were implanted with testosterone 2 weeks after recovery for 6 weeks. For chronic testosterone administration, a 19-mm-long silastic tubing (Dow Corning Corporation, Auburn, MI; 0.062 in. ID; 9.125 in. OD) containing $10 \mathrm{mg}$ of testosterone propionate (Sigma-Aldrich, St. Louis, MO, USA) was implanted subcutaneously at the back of the rat shoulder, following the method as described by Reckelhoff et al. (36). Non-testosterone-treated rats were implanted with empty silastic tubing. The tubings were replaced every 3 weeks. When animals achieved 16 weeks of age, they were subjected for the procedures. Four experimental groups per strain were designed as follows:

Group 1: Sham-operated, intact male WKY and SHR rats

Group 2: Orchidectomized (ORX) male WKY and SHR rats

Group 3: Ovariectomized (OVX) female WKY and SHR rats

Group 4: Ovariectomized (OVX) and testosterone-treated (T) female WKY and SHR rats

\section{In vivo blood pressure measurement}

Rats were anesthetized at 16 weeks of age by pentobarbital sodium anesthesia $(60 \mathrm{mg} / \mathrm{kg}$; Sigma-Aldrich), which was injected intraperitoneally and the dose was maintained throughout the period of anesthesia. Cannulation of the carotid artery follows the method as described by Parasuraman and Raveendran (34). In brief, a small incision was made in the trachea to insert a tracheostomy tube and a cannula pre-filled with heparinized normal saline $(0.5 \mathrm{IU} / \mathrm{ml})$ was then inserted into the artery. The cannula was connected to a PowerLab Data acquisition system (ADInstruments, Australia), which then directly monitors the blood pressure. Blood pressure was recorded after the rats were stabilized at least for $10 \mathrm{~min}$. Data were analyzed by LabChart version 6.0 where the average systolic blood pressure (SBP) and diastolic blood pressure (DBP) were obtained. MAP was then calculated.

\section{Measurement of plasma testosterone level}

At the end of cannulation, blood was collected from the artery and placed into a chilled heparinized tubes. Blood plasma was obtained by centrifuging at $3,000 \times g$ for $20 \mathrm{~min}$ at $4{ }^{\circ} \mathrm{C}$. Plasma testosterone levels were measured using enzyme-linked immunosorbent assay kit (Enzo Life Sciences, Farmingdale, NY, USA) (Cat No. ADI-900-065), following the manufacturer's guidelines. Each assay was performed in triplicate with the lowest sensitivity limit of $5.67 \mathrm{pg} / \mathrm{ml}$.

\section{Statistical analysis}

All data were presented as mean \pm standard error of mean (SEM). All statistical analyses were performed using GraphPad Prism (GraphPad Software Inc., San Diego, CA, USA). Statistical differences between groups were evaluated using independent unpaired Student's $t$-test. Comparisons between more than two groups were performed using one-way analysis of variance with Tukey's post-hoc test. Differences were considered as statistically significant at $P$ level $<0.05$. 


\section{Results}

Effects of gonadectomy on MAP and plasma testosterone level in males and female rats In sham-operated male rats, MAP was higher than sham-operated female rats for both rat strains $(P<0.001)$ (Fig. 1a). Orchidectomy caused a decrease in MAP in male rats $(P<0.01$ for WKY rats and $P<0.001$ for SHR rats). In female rats, ovariectomies also caused a decrease in MAP $(P<0.05$ for WKY rats and $P<0.01$ for SHR rats).

The highest plasma testosterone levels were observed in sham-operated male SHR rats (Fig. 1b). Orchidectomy decreased the plasma testosterone level in both male WKY $(P<0.001)$ and SHR $(P<0.05)$ rats. Similarly, ovariectomy also decreased the plasma testosterone level in female rats of both strains $(P<0.05)$.

Changes in MAP and plasma testosterone levels at different stages of the rat estrous cycle No differences in MAP were reported across the estrous cycle stages for any of the WKY and SHR female rats (Fig. 2a). Similarly, plasma testosterone levels were not significantly different between stages of the estrous cycle in any of the rat strains (Fig. 2b).

Effects of testosterone on MAP and plasma testosterone levels in female rats

In ovariectomized female rats, 6-week treatment with testosterone caused the MAP to increase $(P<0.05$ for WKY rats and $P<0.01$ for SHR rats) (Fig. 3a). In concordant, plasma
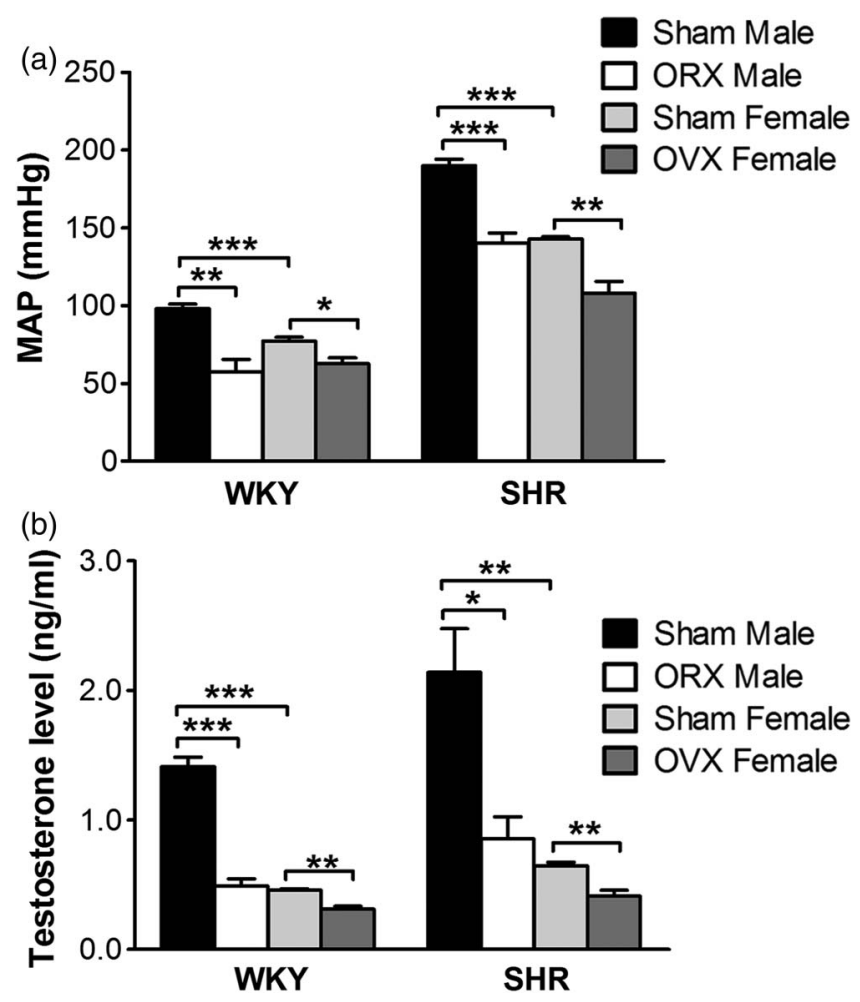

Fig. 1. (a) MAP of 16-week-old WKY and SHR male and female rats as measured by cannulation of the carotid artery. Sham operation and gonadectomies were carried out at 8 weeks of age. (b) Plasma testosterone levels were measured using the blood collected from the carotid artery at the end of the experiment. Data were presented as mean $\pm \mathrm{SEM} ; n=5$ /group; ${ }^{*} P<0.05 ; * * P<0.01$

$* * * P<0.001$. Sham: shamoperated; ORX: orchidectomized; OVX: ovariectomized 



Fig. 2. (a) MAP of 16-week-old naive female WKY and SHR rats as measured by cannulation of the carotid artery. The stages of estrous cycle were determined by vaginal smears. (b) Plasma testosterone levels were measured using the blood collected from the carotid artery at the end of the experiment. Data were presented as mean \pm SEM; $n=5-8$ /group.

Ps: proestrus; Es: estrus; Ms: metetrus; Ds: diestrus

testosterone levels in female rats receiving testosterone treatment markedly increased $(P<0.05)$ (Fig. 3b).

\section{Discussion}

Sex steroids are known to play an important role in the blood pressure regulation in males and females $(19,21,28)$. In this study, a positive correlation between plasma testosterone level and MAP has been identified. MAP was found to be higher in intact male WKY and SHR rats as compared with intact female WKY and SHR rats and these findings were consistent with the reports by others $(4,7,30,31,35)$. In this study, gonadectomies performed prior to pubertal age, that is, 8 weeks were found to decrease the MAP in both male and female normotensive WKY and SHR rats. These findings were also consistent with the report by Masubuchi et al. (31) who showed that the blood pressure in adult male and female rats could be affected by gonadectomies. In male rats, it was found that orchidectomy caused a decrease in the levels of endogenous testosterone, whereas in female rats, ovariectomy caused a decrease in endogenous testosterone levels.

Changes in plasma testosterone levels were found to positively correlate with MAP, as seen in male and female intact and gonadectomized rats and in gonadectomized female rats receiving exogenous testosterone treatment. The increase in MAP in ovariectomized female 


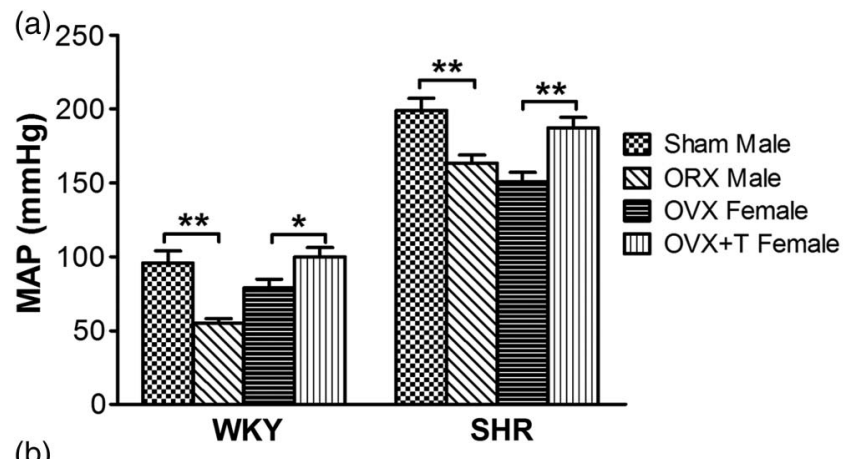

(b)

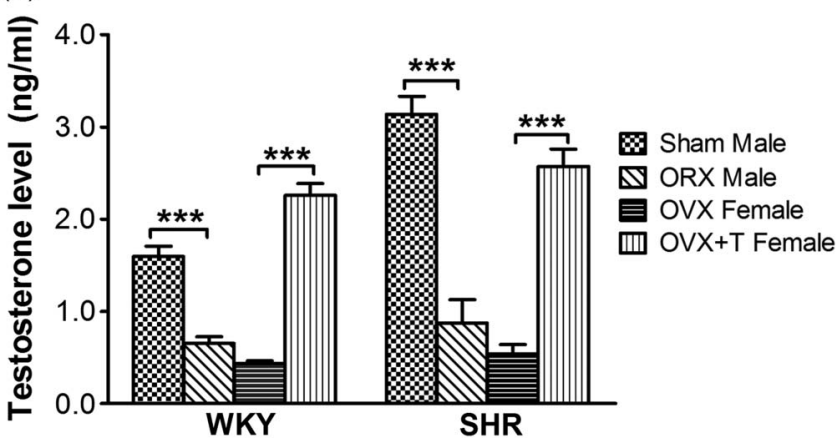

Fig. 3. (a) MAP of 16-week-old WKY and SHR male and female rats as measured by cannulation of the carotid artery. Sham operation and gonadectomies were performed at 8 weeks of age. At the age of 10 weeks, female rats were treated with testosterone implants for 6 weeks. (b) Plasma testosterone levels were measured using the blood collected from the carotid artery at the end of the experiment. Data were presented as mean \pm SEM; $n=5$ /group; $* P<0.05 ; * * P<0.01$ $* * * P<0.001$. Sham: shamoperated; ORX: orchidectomized; OVX: ovariectomized; T: testosterone

rats of both strains following exogenous testosterone treatment strengthened the argument that testosterone increases the blood pressure as this model eliminates the effect of male sex chromosome, which has been implicated in causing higher blood pressure in males compared with females $(13,14,32)$. Our findings were supported by the results of Reckelhoff et al. (36) who demonstrated an increase in MAP in testosterone-treated female SHR rats. In this study, increase in MAP was also seen in testosterone-treated normotensive WKY rats, indicating that testosterone-induced increase in blood pressure could also happen in the normotensive condition. The findings in male WKY and SHR rats in which orchidectomy had also caused a decrease in blood pressure have further strengthened the view that testosterone is involved in increasing the blood pressure, a finding similar to that found by others (23).

There were conflicting reports with regard to the effect of ovariectomy on MAP $(9,35$, 42). Ovariectomy has been reported not to cause changes in blood pressure of wild-type mice. In another study using Sprague-Dawley (SD) rats at 10-12 weeks of age, no difference in the MAP between intact and ovariectomized female rats was found (49). In another study, Crofton and Share (6) have shown that in female SD rats, gonadectomy did not cause significant changes in blood pressure when compared with intact rats. Their study further showed that gonadectomized female rats were more sensitive toward deoxycorticosteronesalt induced increase in the blood pressure. Ovariectomy performed in 6-week-old female Dahl salt-sensitive rats which were given low-sodium diet did not cause any significant changes in blood pressure at 12 weeks ( 3 months), however at 16 weeks ( 4 months), the SBP in these rats was significantly higher than in intact rats, and these differences persisted up to the age of 84 weeks (12 months) (20). 
In contrast, our findings and several other findings indicated that ovariectomy reduced the blood pressure in female rats. Stachowiak et al. (44) reported that 28 days after ovariectomy, the blood pressure in female rats markedly decreased which was associated with a marked atrophy of the adrenal cortices. A recent study also indicated that there was a trend of a reduction of the blood pressure taken at 24 weeks of age in female SD rats where ovariectomy was performed at 7 weeks of age (5). A study conducted in 12-week-old Wistar rats indicated that there was a transient decrease in MAP measured using a tail cuff, 2 weeks after ovariectomy which subsequently returned to pre-ovariectomized values (25). In this study, we measured the blood pressure in anesthetized female rats via carotid artery cannulation which showed similar results.

We hypothesized that the decrease in MAP following ovariectomy in female WKY and SHR rats may be, at least partly, mediated by suppression of adrenal secretory activity, due to the lack of circulating estrogens, which are known to stimulate the hypophyseal adrenocorticotropic hormone release. Estrogen has been reported to be able to increase the renninangiotensin system activity $(33,41)$. Therefore, a decrease in estrogen levels following removal of endogenous source of estrogen via ovariectomy will result in the alleviation of estrogen-induced increase in renin-angiotensin-aldosterone system activity, contributing to the reduction in blood pressure.

This study has shown that there was no significant difference in MAP during the rats' estrous cycle. Previous studies have also demonstrated that blood pressure did not change throughout the estrous cycle in rodents $(2,11)$. Liu and Ely (27) also reported that the blood pressure in female SHR rats did not change throughout the estrous cycle, a finding consistent with ours. The fact that there were no changes in blood pressure observed throughout the estrous cycle indicated that fluctuation in endogenous sex-steroid levels throughout the cycle had no influence on the blood pressure. Despite the high estrogen levels at estrus and high progesterone levels at diestrus, these hormones seem not to have an influence on the blood pressure. On the other hand, plasma testosterone levels did not change significantly throughout the estrous cycle (40). Therefore, it can be concluded that in intact female rats, the blood pressure changes rather depend on other factors than the fluctuating levels of sexsteroid hormones.

This study has several limitations. The blood pressure measurements made in anesthetized rats might not reflect the changes in blood pressure of conscious animals. Future studies should be performed using a radio-telemetry device that can provide a real-time blood pressure monitoring in conscious, freely moving animals (1). In conclusion, this study may have implications in the understanding of gender difference in blood pressure regulation. Testosterone might be implicated in causing higher incidence of hypertension in men, and in women after menopause (38). The elevated MAP in post-menopausal women could partly be related to the elevated levels of plasma testosterone, in addition to the falling levels of plasma estrogen $(43,50)$.

\section{Acknowledgements}

This study was supported by University of Malaya Research Grants (UMRG) RP011-13HTM and Postgraduate Research Fund (PPP) PG002-2014B. The authors would like to thank the Division of Laboratory Medicine, University Malaya Medical Centre (UMMC) for the blood hormone analysis.

\section{Conflict of interest}

The authors declare no conflict of interest. 


\section{REFERENCES}

1. Alam MA, Parks C, Mancarella S: Long-term blood pressure measurement in freely moving mice using telemetry. J. Vis. Exp. (111), e53991 (2016). https://www.jove.com/video/53991/long-term-blood-pressuremeasurement-freely-moving-mice-using

2. Capone C, Anrather J, Milner TA, Iadecola C: Estrous cycle dependent neurovascular dysfunction induced by angiotensin II in the mouse neocortex. Hypertension 54(2), 302-307 (2009)

3. Chen M-J, Yang W-S, Yang J-H, Chen C-L, Ho H-N, Yang Y-S: Relationship between androgen levels and blood pressure in young women with polycystic ovary syndrome. Hypertension 49(6), 1442-1447 (2007)

4. Chen YF, Meng QC: Sexual dimorphism of blood pressure in spontaneously hypertensive rats is androgen dependent. Life Sci. 48(1), 85-96 (1991)

5. Chinnathambi V, Balakrishnan M, Yallampalli C, Sathishkumar K: Prenatal testosterone exposure leads to hypertension that is gonadal hormone-dependent in adult rat male and female offspring. Biol. Reprod. 86(5), 137 (2012)

6. Crofton JT, Share L: Gonadal hormones modulate deoxycorticosterone-salt hypertension in male and female rats. Hypertension 29(1), 494-499 (1997)

7. Dalpiaz PLM, Lamas AZ, Caliman IF, Ribeiro RF, Abreu GR, Moyses MR, Andrade TU, Gouvea SA, Alves MF, Carmona AK, Bissoli NS: Sex hormones promote opposite effects on ACE and ACE2 activity, hypertrophy and cardiac contractility in spontaneously hypertensive rats. PLoS One 10(5), e0127515 (2015)

8. Davis DD, Ruiz AL, Yanes LL, Iliescu R, Yuan K, Moulana M, Racusen LC, Reckelhoff JF: Testosterone supplementation in male obese Zucker rats reduces body weight and improves insulin sensitivity, but increases blood pressure. Hypertension 59(3), 726-731 (2012)

9. De Melo VU, Saldanha RRM, Dos Santos CR, De Campos Cruz J, Lira VA, Santana-Filho VJ, Michelini LC: Ovarian hormone deprivation reduces oxytocin expression in paraventricular nucleus preautonomic neurons and correlates with baroreflex impairment in rats. Front. Physiol. 7, 461 (2016)

10. Dubey RK, Oparil S, Imthurn B, Jackson EK: Sex hormones and hypertension. Cardiovasc. Res. 53(3), 688-708 (2002)

11. Ebine T, Toriumi H, Shimizu T, Unekawa M, Takizawa T, Kayama Y, Shibata M, Suzuki N: Alterations in the threshold of the potassium concentration to evoke cortical spreading depression during the natural estrous cycle in mice. Neurosci. Res. 112, 57-62 (2016)

12. Echiburú B, Crisosto N, Maliqueo M, Pérez-Bravo F, de Guevara AL, Hernández P, Cavada G, Rivas C, Clavel A, Sir-Petermann T: Metabolic profile in women with polycystic ovary syndrome across adult life. Metabolism 65(5), 776-782 (2016)

13. Ellis JA, Stebbing M, Harrap SB: Association of the human Y chromosome with high blood pressure in the general population. Hypertension 36(5), 731-733 (2000)

14. Ely D, Underwood A, Dunphy G, Boehme S, Turner M, Milsted A: Review of the Y chromosome, Sry and hypertension. Steroids 75(11), 747-753 (2010)

15. Filgueira FP, Lobato NS, DosSantos RA, Oliveira MA, Akamine EH, Tostes RC, Fortes ZB, Carvalho MH: Endogenous testosterone increases leukocyte-endothelial cell interaction in spontaneously hypertensive rats. Life Sci. 90(17-18), 689-694 (2012)

16. Fogari R, Preti P, Zoppi A, Fogari E, Rinaldi A, Corradi L, Mugellini A: Serum testosterone levels and arterial blood pressure in the elderly. Hypertens. Res. 28(8), 625-630 (2005)

17. Ganten U, Schroder G, Witt M, Zimmermann F, Ganten D, Stock G: Sexual dimorphism of blood pressure in spontaneously hypertensive rats: effects of anti-androgen treatment. J. Hypertens. 7(9), 721-726 (1989)

18. Goldman RK, Azar AS, Mulvaney JM, Hinojosa-Laborde C, Haywood JR, Brooks VL: Baroreflex sensitivity varies during the rat estrous cycle: role of gonadal steroids. Am. J. Physiol. Regul. Integr. Comp. Physiol. 296(5), R1419-R1426 (2009)

19. Hay M: Sex, the brain and hypertension: brain oestrogen receptors and high blood pressure risk factors. Clin. Sci. (Lond.) 130(1), 9-18 (2016)

20. Hinojosa-Laborde C, Craig T, Zheng W, Ji H, Haywood JR, Sandberg K: Ovariectomy augments hypertension in aging female Dahl salt-sensitive rats. Hypertension 44(4), 405-409 (2004)

21. Huang CK, Lee SO, Chang E, Pang H, Chang C: Androgen receptor (AR) in cardiovascular diseases. J. Endocrinol. 229(1), R1-R16 (2016)

22. Hughes GS, Mathur RS, Margolius HS: Sex steroid hormones are altered in essential hypertension. J. Hypertens. 7(3), 181-187 (1989) 
23. Jenkins C, Salisbury R, Ely D: Castration lowers and testosterone restores blood pressure in several rat strains on high sodium diets. Clin. Exp. Hypertens. 16(5), 611-625 (1994)

24. Khaw KT, Barrett-Connor E: Blood pressure and endogenous testosterone in men: an inverse relationship. J. Hypertens. 6(4), 329-332 (1988)

25. Lauda ski K, Cudnoch-J drzejewska A: Effects of ovariectomy on the regulation of cardiovascular functions in female Wistar rats. Med. Sci. Monit. 7(6), BR1188-BR1192 (2001)

26. Linton L, Taylor M, Dunn S, Martin L, Chavez S, Stanitz G, Huszti E, Minkin S, Boyd N: Associations of serum levels of sex hormones in follicular and luteal phases of the menstrual cycle with breast tissue characteristics in young women. PLoS One 11(10), e0163865 (2016)

27. Liu B, Ely D: Testosterone increases: sodium reabsorption, blood pressure, and renal pathology in female spontaneously hypertensive rats on a high sodium diet. Adv. Pharmacol. Sci. 2011, 817835 (2011)

28. Maranon R, Reckelhoff JF: Sex and gender differences in control of blood pressure. Clin. Sci. (Lond.) 125(7), 311-318 (2013)

29. Marcondes FK, Bianchi FJ, Tanno AP: Determination of the estrous cycle phases of rats: some helpful considerations. Braz. J. Biol. 62, 609-614 (2002)

30. Maris ME, Melchert RB, Joseph J, Kennedy RH: Gender differences in blood pressure and heart rate in spontaneously hypertensive and Wistar-Kyoto rats. Clin. Exp. Pharmacol. Physiol. 32(1-2), 35-39 (2005)

31. Masubuchi Y, Kumai T, Uematsu A, Komoriyama K, Hirai M: Gonadectomy-induced reduction of blood pressure in adult spontaneously hypertensive rats. Acta Endocrinol. (Copenh.) 101(1), 154-160 (1982)

32. Molina E, Clarence EM, Ahmady F, Chew GS, Charchar FJ: Coronary artery disease: why we should consider the Y chromosome. Heart Lung Circ. 25(8), 791-801 (2016)

33. O'Hagan TS, Wharton W, Kehoe PG: Interactions between oestrogen and the renin angiotensin system - potential mechanisms for gender differences in Alzheimer's disease. Am. J. Neurodegener. Dis. 1(3), 266-279 (2012)

34. Parasuraman S, Raveendran R: Measurement of invasive blood pressure in rats. J. Pharmacol. Pharmacother. 3(2), 172-177 (2012)

35. Pijacka W, Clifford B, Walas D, Tilburgs C, Joles JA, McMullen S, Langley-Evans SC: Impact of gonadectomy on blood pressure regulation in ageing male and female rats. Biol. Sex Differ. 7, 64 (2016)

36. Reckelhoff JF, Zhang H, Granger JP: Testosterone exacerbates hypertension and reduces pressure-natriuresis in male spontaneously hypertensive rats. Hypertension 31(1 Pt. 2), 435-439 (1998)

37. Reckelhoff JF, Zhang H, Srivastava K: Gender differences in development of hypertension in spontaneously hypertensive rats: role of the renin-angiotensin system. Hypertension 35(1), 480-483 (2000)

38. Regitz-Zagrosek V: Sex and gender differences in health: science and society series on sex and science. EMBO Rep. 13(7), 596-603 (2012)

39. Rothman MS, Carlson NE, Xu M, Wang C, Swerdloff R, Lee P, Goh VHH, Ridgway EC, Wierman ME: Reexamination of testosterone, dihydrotestosterone, estradiol and estrone levels across the menstrual cycle and in postmenopausal women measured by liquid chromatography-tandem mass spectrometry. Steroids $76(1-2)$, 177-182 (2011)

40. Rush ME, Blake CA: Serum testosterone concentrations during the 4-day estrous cycle in normal and adrenalectomized rats. Exp. Biol. Med. 169(2), 216-221 (1982)

41. Safari T, Nematbakhsh M, Evans RG, Denton KM: High-dose estradiol-replacement therapy enhances the renal vascular response to angiotensin II via an AT2-receptor dependent mechanism. Adv. Pharmacol. Sci. 2015, 682745 (2015)

42. Sandberg K, Ji H: Sex differences in primary hypertension. Biol. Sex Differ. 3, 7 (2012)

43. Secreto G, Sieri S, Agnoli C, Grioni S, Muti P, Zumoff B, Sant M, Meneghini E, Krogh V: A novel approach to breast cancer prevention: reducing excessive ovarian androgen production in elderly women. Breast Cancer Res. Treat. 158(3), 553-561 (2016)

44. Stachowiak A, Nussdorfer GG, Malendowicz L: Ovariectomy-induced changes in the adrenal cortex of spontaneously hypertensive rats. Histol. Histopathol. 6(2), 257-259 (1991)

45. Svartberg J, von Muhlen D, Schirmer H, Barrett-Connor E, Sundfjord J, Jorde R: Association of endogenous testosterone with blood pressure and left ventricular mass in men. The Tromsø Study. Eur. J. Endocrinol. 150(1), 65-71 (2004)

46. Takezawa H, Hayashi H, Sano H, Ebihara S: Estrous-related variations of blood pressure and heart rate in female rats. Front. Med. Biol. Eng. 6(2), 131-137 (1994)

47. Vlachopoulos C, Pietri P, Ioakeimidis N, Aggelis A, Terentes-Printzios D, Abdelrasoul M, Gourgouli I, Stefanadis C, Tousoulis D: Inverse association of total testosterone with central haemodynamics and left ventricular mass in hypertensive men. Atherosclerosis 250, 57-62 (2016) 
48. Wenner MM, Stachenfeld NS: Blood pressure and water regulation: understanding sex hormone effects within and between men and women. J. Physiol. 590(23), 5949-5961 (2012)

49. Xue B, Badaue-Passos D, Guo F, Gomez-Sanchez CE, Hay M, Johnson AK: Sex differences and central protective effect of $17 \beta$-estradiol in the development of aldosterone $/ \mathrm{NaCl}$-induced hypertension. Am. J. Physiol. Heart Circ. Physiol. 296(5), H1577-H1585 (2009)

50. Yasui T, Matsui S, Tani A, Kunimi K, Yamamoto S, Irahara M: Androgen in postmenopausal women. J. Med. Invest. 59(1-2), 12-27 (2012) 